\title{
A new species of Pyropia (Rhodophyta, Bangiaceae), from the Pacific coast of Mexico, based on morphological and molecular evidence
}

\author{
LUZ ELENA MATEO-CID ${ }^{1 *}$, ANGELA CATALINA MENDOZA-GONZÁLEZ ${ }^{1}$, JHOANA DÍAZ- \\ LARREA $^{2}$, ABEL SENTÍES $^{2}$, FRANCISCO F. PEDROCHE ${ }^{3} \&$ JUAN DIEGO SÁNCHEZ HEREDIA $^{4}$ \\ ${ }^{1}$ Departamento de Botánica, Escuela Nacional de Ciencias Biológicas, IPN. Carpio y Plan de Ayala s/n. Mexico, D.F. 11340. \\ ${ }^{2}$ Departamento de Hidrobiología. Universidad Autónoma Metropolitana-Iztapalapa. A.P. \\ 55-535, Mexico, D.F. 09340, Mexico. \\ ${ }^{3}$ Departamento de Ciencias Ambientales. Universidad Autónoma Metropolitana-Lerma, Mexico. \\ ${ }^{4}$ Facultad de Biología. Universidad Michoacana de San Nicolás de Hidalgo, Morelia, Michoacán, Mexico. \\ *Corresponding author: E-mail: Imateoc@ipn.mx
}

\begin{abstract}
Pyropia raulaguilarii sp. nov. is described from Michoacán, tropical Mexican Pacific, on basis of comparative morphology and nrSSU, $r b c \mathrm{~L}$ sequence analysis. It is distinguished from other Pyropia species by the foliose and lanceolate gametophyte, a monoecious thallus and the zygotosporangia in packets of $2 \times 2 \times 4$. The phylogenetic analysis showed that the two Pacific Mexican samples, from Caletilla and Carrizalillo (Michoacán), were almost identical and formed a distinctive and well supported clade segregated from other species of Pyropia from Brazil, USA and Mexico. The Mexican entity is morphologically and genetically distinct from other Pyropia species, suggesting that this species should be assigned to a new taxon.
\end{abstract}

Key words: Bangiales, molecular phylogeny, $\mathrm{nrSSU}, r b c \mathrm{~L}$, marine red algae.

\section{Introduction}

Species of Porphyra C.Agardh have few characters for distinguishing species, however, these characters alone have proved to be misleading based on the discovery, using molecular sequences, of many cryptic taxa among species with very similar morphologies (e.g. Brodie \& Irvine 1997, Broom et al. 2002, 2004, Neefus et al. 2002, Teasdale et al. 2002, Lindstrom \& Fredericq 2003, Niwa et al. 2005, Brodie et al. 2007, Lindstrom 2008). Almost all of these studies have used either nrSSU or plastid $r b c \mathrm{~L}$ gene. The use of the more conservative nrSSU locus has generally resulted in phylogenies with a well-supported "backbone" but little differentiation of closely related taxa. The protein-coding $r b c \mathrm{~L}$ gene clearly distinguishes species, but support for ancient divergences is often less than the one obtained in analyses based on the nrSSU gene (Lindstrom \& Fredericq 2003, Nelson et al. 2006).

"Porphyra sensu lato" as the cited papers include species no longer considered part of Porphyra (sensu stricto) (Sutherland et al. 2011). Molecular studies have revealed extensive cryptic speciation within the genus Porphyra (Broom et al. 1999, 2004, 2010, Jones et al. 2004) and has been used in the genetics (Kuang et al. 1998) and breeding of Porphyra species since the end of the twentieth century (Jia et al. 2000, Xu et al. 2011). Most previous reports were focused on the identification, protection, construction, and classification of different strains (Iitsuka et al. 2002, Kunimoto et al. 2003, Liu et al. 2004, Sun et al. 2005, Weng et al. 2005, Qiao et al. 2007). 
Recently, Sutherland et al. (2011) carried out an important revision that constitutes a major change in understanding relationships and evolution in Bangiales, and combined analyses of the nuclear SSU rRNA and chloroplast $r b c \mathrm{~L}$ regions recognizing five new genera and two others that were resurrected. The genus Porphyra is now restricted to five described species and a number of undescribed species. Other foliose taxa previously placed in Porphyra are now recognized to belong to the genera Boreophyllum S.C.Lindstr. et al., Clymene W.A.Nelson, Fuscifolium S.C.Lindstr., Lysithea W.A.Nelson, Miuraea Kukuchi et al., Pyropia J.Agardh and Wildemania De Toni.

The genus Pyropia is characterized by a) gametophytic thalli monostromatic, linear, ovate, orbicular or funnel shaped, b) margins entire or dentate, planar, undulate, or ruffled, c) color variable, d) blades sessile or with brief stipe, e) vegetative cells embedded in colorless, firm gelatinous matrix, f) cells with one or two stellate chloroplasts, each one with central pyrenoid, g) plants monoecius or dioecius, h) sexual thalli monoecious with groups of cells forming either spermatangia and zygotosporangia, these often in streaks or rectangular patches, or divided into separate male and female sectors by a vertical or horizontal line, i) in dioecious thalli, the spermatangia and zygotosporangia forming as continuous areas along the margins of the blade, and $\mathrm{j}$ ) the conchocelis phase is a microscopic and filamentous stage (Sutherland et al. 2011).

During recent research on the diversity of benthic marine algae of the state of Michoacán, tropical Pacific Mexico coast, an undescribed red alga was collected. This entity is characterized by thalli with monostromatic blades, arising from a single discoid attachment, and sexual thalli monoecious with spermatangia and zygotosporangia in patches. This internal organization corresponds to the genus Pyropia.

\section{Materials \& Methods}

Morphological analysis. Thalli of Pyropia were observed at two localities in southern Michoacán: Caletilla and El Carrizalillo. Slides of fertile specimens were prepared for microscopic observation from material preserved in 5\% formalin-seawater. Whole mount slides with cross and transverse sections were made by hand with razor blades, these sections were stained in $1 \%$ aqueous aniline blue, acidified by the addition of $1 \mathrm{~N}$ $\mathrm{HCl}$, according to Mateo-Cid et al. (2005) and permanently mounted on microscope slides with $70 \%$ Karo Syrup ${ }^{\circledR}$ with $1 \%$ phenol added as a preservative. Photomicrographs were taken with a Sony MPEGMOVIEVX (Tokyo, Japan) coupled to an Olympus CX31 Microscope (Manila, Filipinas). Images of specimens were taken with a digital SONY camera (MPEGMOVIEVX). Digital images were edited and assembled on plates using Adobe Photoshop 7.0 (San Jose, CA, USA).

Molecular analysis. Samples used for molecular analysis were dried in silica gel. Total DNA was extracted using the DNeasy Plant Mini Kit (Qiagen, Valencia, CA, USA) following the manufacturer's instructions. Sequences of the nrSSU and the $r b c \mathrm{~L}$ genes from Bangiales taxa were obtained. The specimens sequenced in this study, plus other available sequences used for the phylogenetic analysis, are shown in Table 1, including their accession numbers in the National Center for Biotechnology Information (NCBI) GenBank data base.

The $r b c \mathrm{~L}$ and nrSSU regions were amplified with primers suggested by Freshwater \& Rueness (1994) and Saunders et al. (1996), respectively, by using the Taq PCR Core Kit (Quiagen). All polymerase chain reaction (PCR) products were electrophoresed on $1 \%$ agarose gel to verify product size. PCR products were purified with Qiagen QIAquick Purification Kit following the manufacturer's instructions. Sequencing was carried out using PCR primers with the BigDye Terminator Cycle Sequencing Reaction Kit (Applied Biosystems, NJ, USA) on an ABI PRISM 3100 Genetic Analyzer (Applied Biosystems). The authors performed the nucleotide BLAST (Basic Local Alignment Search Tool) of the sequences with the program implemented in the NCBI (National Center for Biotechnology Information) home page. Sequences editing was performed with the Sequence Navigator (Applied Biosystems) and aligned according to the CLUSTAL algorithm (Thompson et al. 1994). 
Phylogenetic analysis. Three data sets were constructed -nrSSU data set, the $r b c \mathrm{~L}$ data set, and a combined data set- with the genes concatenated. Outgroup species (Dione arcuata W.A.Nelson, Minerva aenigmata W.A.Nelson and Miuraea migitae (N. Kikuchi et al.) N. Kikuchi et al. were selected because of a close phylogenetic relationship with the ingroup (Sutherland et al. 2011).

Phylogenetic relationships were inferred with PAUP* 4.0b10 (Swofford 2002) and MrBayes v.3.0 beta 4 (Huelsenbeck \& Ronquist 2001). Maximum-parsimony trees (MP) were constructed using the heuristic search option, tree-bisection-reconnection branch swapping algorithm, unordered and unweight characters, and gaps treated as missing data. Furthermore, distance analysis was conducted under Neighbour-Joining (NJ) algorithm.

Support values for the relationships discovered in analyses were calculated by performing bootstrap analyses (Felsenstein 1985) as implemented in PAUP*. Twenty thousand heuristic search replicates were executed using the TBR branch-swapping algorithm. Consistency (CI) and Homoplasy (HI) indexes were computed to evaluate the level of homoplasy in the most parsimonious tree.

Bayesian trees were constructed for both the single gene data sets and the concatenated data set. The model used was the general-time-reversible model of nucleotide substitution with invariant sites and gamma distributed rates for the variable sites $(G T R+I+G)$ for all three data sets. This model was selected based on maximum likelihood ratio tests implemented by the software Model Test version 3.06 (Posada \& Crandall 1998 ) with a significance level of 0.01 . Analyses were started from random trees, and consisted of two runs, each of four chains (one hot and three cold), of 4 million generations for each data set. The stationary level was reached at generation 17000. Therefore, trees saved until generation 16500 were the 'burn in' of the chain, and inferences about the phylogeny were based on those trees sampled after generation 16500 . To visualize the burn-in of -LnL or model parameters, we use the software Tracer v1.4 (Rambaut \& Drummond 2007). A 50\% consensus tree as implemented by PAUP* was computed after the 'burnin'. The range of $r b c \mathrm{~L}$ divergence values within and among species was calculated using uncorrected ' $p$ ' distances using PAUP*.

\section{Results}

\section{Pyropia raulaguilarii Mateo-Cid, Mendoza-González \& Sentíes, sp. nov. (Figs 1-8, Table 2)}

Gametophytum foliosum, monostromaticum, lanceolatum, basis umbilicalis, margo crispata. Thalli 4 ad $6 \mathrm{~cm}$, adhaerentes perfecte ad papyrum. Thalli steriles usque 40-42 $\mu \mathrm{m}$ crassi. Color rubrum ad aliquando purpureus. Thalli sexuales monoecii spermatangia in viridi inaequalis intermixtam zygotosporangia in rectangulum vel irregularis inaequaliter. Sectiones fertiles (feminei) 50-55 $\mu \mathrm{m}$ crassi. Structurae reproductivae marginales, maculis extensiae intro. Spermatangia in sarcinula $2 \times 2 \times 4 \times 4$. Zygotosporangia in sarcinula $2 \times 2 \times 4$.

Type:-MEXICO. Michoacán: Carrizalillo, low deciduous forest, $18^{\circ} 36^{\prime} 00^{\prime \prime} \mathrm{N}, 102^{\circ} 33^{\prime} 03^{\prime \prime} \mathrm{W}, 0 \mathrm{~m}$ a.s.l., coll. Mendoza-González and Mateo-Cid, 04-04-2009, MICH-09-A/01 (holotype: ENCB!, isotype: UAMIZ!, paratype: Michoacán: Caletilla, $18^{\circ} 03^{\prime} 28^{\prime \prime} \mathrm{N}, 102^{\circ} 42^{\prime} 13^{\prime \prime}$ W, coll. Mendoza González and Sánchez Heredia, 17-05-2006, MICH-06-30/01, ENCB!).

Gametophyte foliose, monostromatic, lanceolate, rarely branched (Fig. 1), often deeply ruffled, margin undulating (Fig. 3). Thalli 4-6 cm high, blades 1-2 cm diameter, red to purple color. Adhering completely to paper. The plants have a well-developed umbilicate base, which is closely adherent to the substratum and attached by rhizoidal cells (Fig. 2). Sterile thalli 40-42 $\mu \mathrm{m}$ thick (Fig. 4), vegetative cells measuring 14-17 $\mu \mathrm{m}$ in diameter and $20-25 \mu \mathrm{m}$ in length. Vegetative cells with a single stellate plastid with one pyrenoid. Reproductive plants: sexual thalli monoecious, spermatangia in green patches intermixed with zygotosporangia in rectangular or irregular patches in fertile regions of the blades. Fertile female sectors 50$55 \mu \mathrm{m}$ thick (Fig. 5). Reproductive structures marginal, extending inward in alternating patches. Submarginal tissue paler than the adjacent outer zygotosporangial and inner sterile tissues. Zygotosporangia in packets of 2 $\times 2 \times 4$ (Figs 7, 8). Spermatangia in packets of $2 \times 2 \times 4 \times 4$ (Fig. 6). 

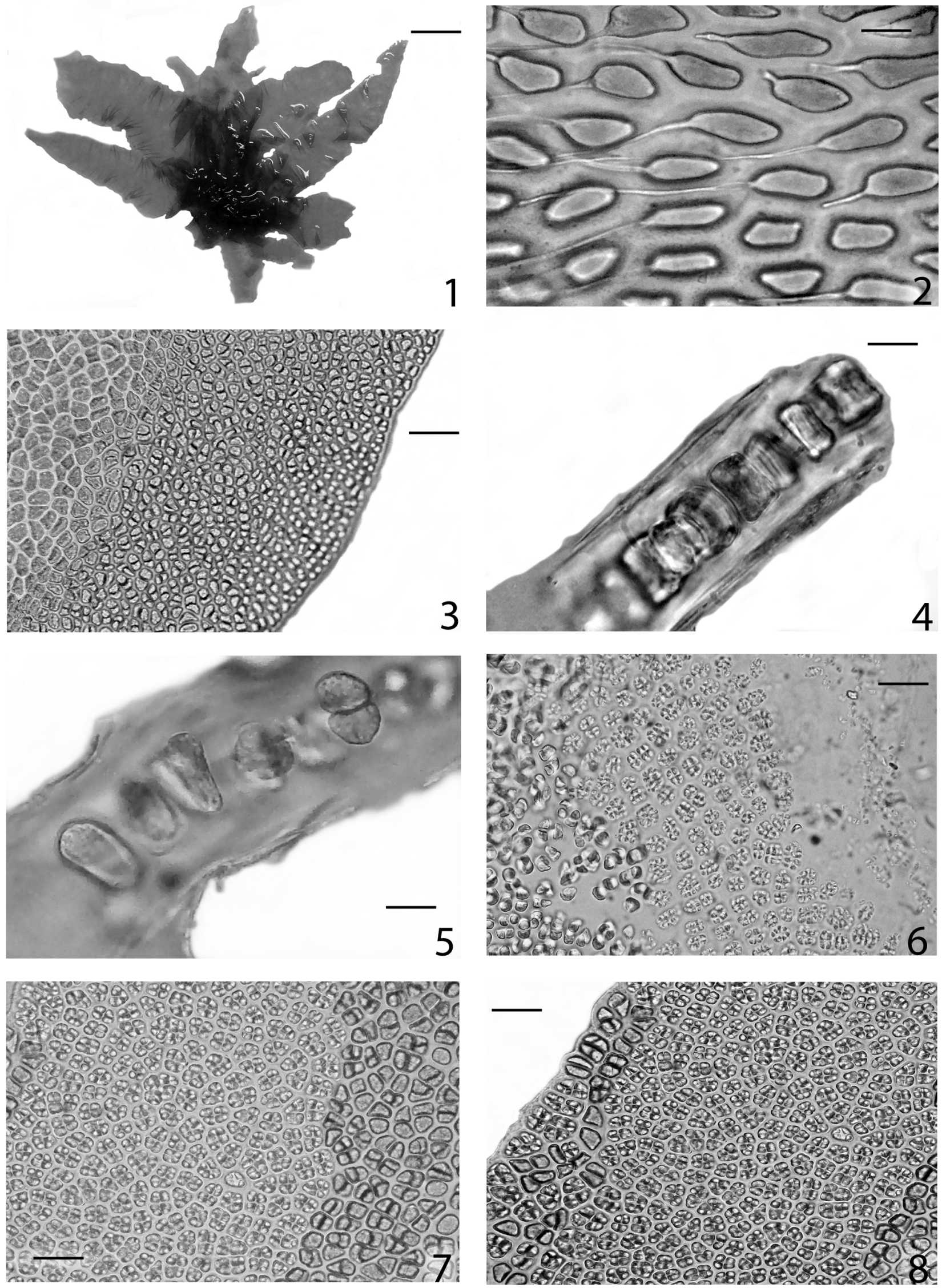

FIGURES 1-8: Pyropia raulaguilarii sp. nov. Fig. 1: Habit of the foliose gametophyte. Holotype. El Carrizalillo (ENCB 19 603). Fig. 2: Rhizoidal cells in surface view. Fig. 3: Margin of thallus in surface view. Fig. 4: Transverse section through thallus showing monostromatic blade. Fig. 5: Transverse section of female portion. Fig. 6: Mature spermatangia in surface view. Fig. 7: Surface view of zygotosporangial region of thallus. Fig. 8: Mature marginal zygotosporangias in surface view. Scale bars: Fig. 1: 12 mm; Figs 2, 6: $30 \mu \mathrm{m}$; Figs 3, 7, 8: $40 \mu \mathrm{m}$; Fig. 4: $15 \mu \mathrm{m}$; Fig. 5: $20 \mu \mathrm{m}$. 
Habitat: -On rocks, intertidal pools.

Etymology: — The species epithet is named in honor of Prof. Raúl Aguilar Rosas, a prominent Mexican phycologist and beloved colleague from Facultad de Ciencias Marinas, Universidad Autónoma de Baja California, Mexico.

Molecular analyses: - Samples of Pyropia raulaguilarii from Carrizalillo and Caletilla (Michoacán, Mexico) diverged each other 0.02-0.03\%. These samples diverged 3.0 to 3.4\% from the GenBank sequence called Pyropia $c f$. thuretii from Mazatlán, Mexico (Table 1). The concatenated phylogenetic data set consisted of 62 taxa (Table 1), including 59 ingroup and three outgroup taxa, with 2767 characters: 1300 from the nrSSU and 1467 from the $r b c \mathrm{~L}$ gene. Bayesian, parsimony (not shown) and distance (not shown) analyses resulted in similar trees. The topology of the Bayesian tree showed eight lineage corresponding to representatives of the following Bangiales genera: Bangia Lyngbye, Boreophyllum, Clymene, Fuscifolium, Lysithea, Porphyra C.Agardh, Pyropia and Wildemania (Fig. 9). The Pacific Mexican samples processed in the present study were located in two well-supported terminal Pyropia sister clades. The samples collected in Carrizalillo and Caletilla (Michoacán) formed a high supported monophyletic clade with a sister clade called Pyropia cf. thuretii from Mazatlán, Mexico (Table 1).

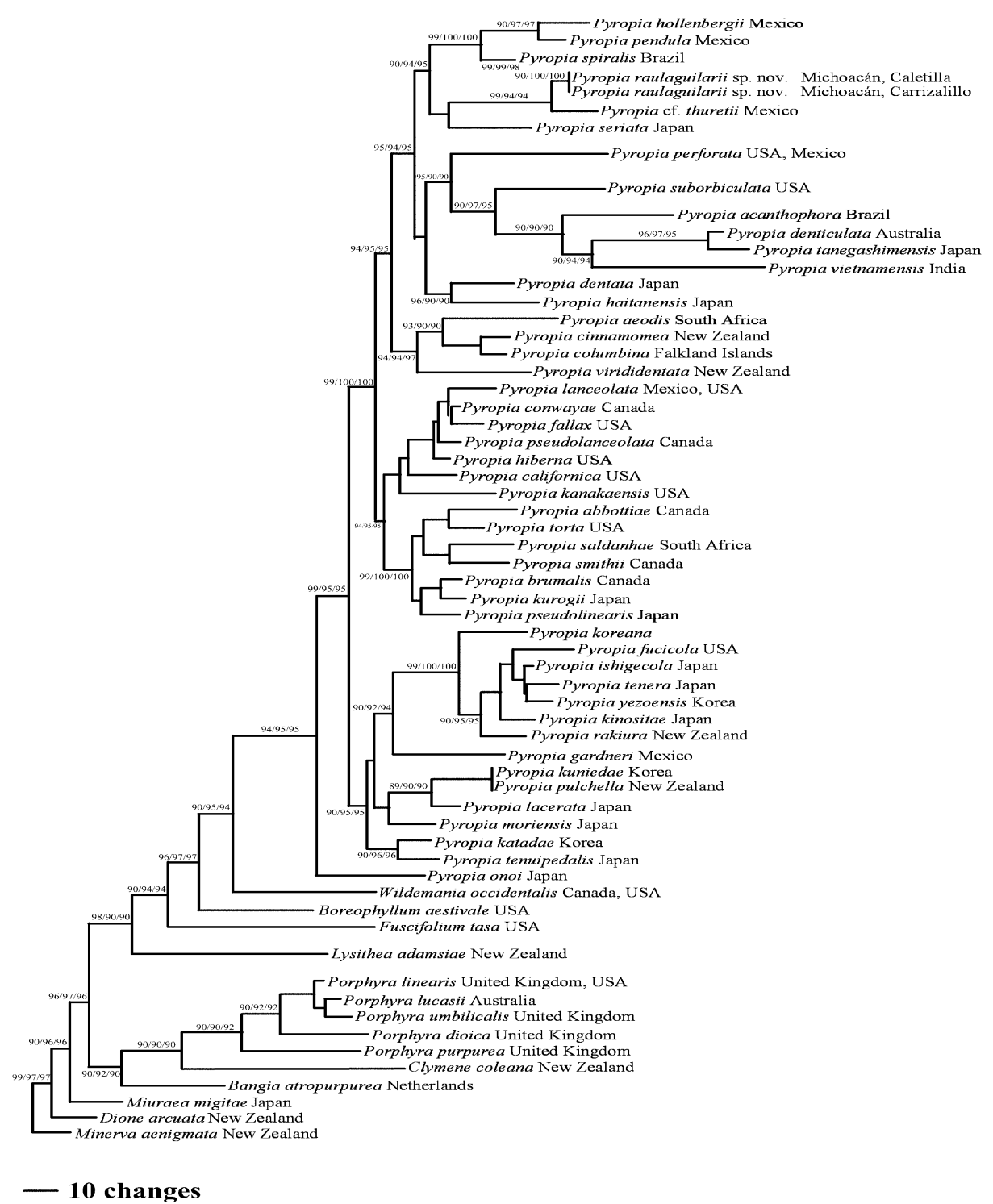

FIGURE 9: Bayesian result for concatenated nuclear SSU ribosomal RNA (nrSSU) and $r b c \mathrm{~L}$ data set. Support values are Bayesian posterior probabilities, and parsimony and distance bootstrap values, respectively. Some internal support values are omitted for clarity. 
TABLE 1: Specimen collection information, voucher numbers and GenBank accession numbers of sequences used in phylogenetic analysis.

\begin{tabular}{|c|c|c|c|}
\hline \multirow[t]{2}{*}{ Taxon } & \multirow[t]{2}{*}{ Collection data } & \multicolumn{2}{|c|}{ GenBank Accession Numbers } \\
\hline & & nrSSU & $r b c \mathrm{~L}$ \\
\hline Bangia atropurpurea (Roth) C.Agardh & Netherlands, Ysselmeer, Enschede. & AF169341 & AF169330 \\
\hline $\begin{array}{l}\text { Boreophyllum aestivale (S.C. Lindstr. \& } \\
\text { Fredericq) S.C.Lindstr. }\end{array}$ & $\begin{array}{l}\text { USA, Alaska, Amaknak Island, Captains Bay, } 4 \\
\text { August 2004, UBC A86208 (nrSSU); SCL } 1325 \\
(r b c L) \text {. }\end{array}$ & GU319836 & EU223033 \\
\hline $\begin{array}{l}\text { Clymene coleana (W.A.Nelson) } \\
\text { W.A.Nelson }\end{array}$ & $\begin{array}{l}\text { New Zealand, North I, Leigh, WELT A22181 } \\
\text { (nrSSU); WELT A22181 (rbcL). }\end{array}$ & AF136423 & FJ263672 \\
\hline Dione arcuata $^{\mathrm{b}}$ & $\begin{array}{l}\text { New Zealand, South I, Kaikoura, Ohau Stream, } \\
\text { WELT A23126 (nrSSU); WELT A23126 (rbcL). }\end{array}$ & AY465354 & EU570052 \\
\hline Fuscifolium tasa (Yendo) S.C.Lindstr. & $\begin{array}{l}\text { USA, Alaska, Unalaska Island, Spray Cape, } 4 \text { June } \\
\text { 2005, UBC A86511 (nrSSU); SCL } 12178 \text { ( } r b c \mathrm{~L}) \text {. }\end{array}$ & GU319862 & EU223226 \\
\hline $\begin{array}{l}\text { Lysithea adamsiae (W.A.Nelson) } \\
\text { W.A.Nelson }\end{array}$ & $\begin{array}{l}\text { New Zealand, Antipodes Islands, Orde Lees I, } \\
\text { WELT A023233 (nrSSU); WELT A023233 } \\
(r b c \text { L). }\end{array}$ & HQ687566 & HQ687515 \\
\hline Minerva aenigmata W.A. Nelson ${ }^{\text {b }}$ & $\begin{array}{l}\text { New Zealand, South Island, Westland, Cobden } \\
\text { (nrSSU); New Zealand, North I, Puheke, WELT } \\
\text { A25775 (rbcL). }\end{array}$ & AY184347 & EU570053 \\
\hline Miuraea migitae ${ }^{\mathrm{b}}$ & Japan, Osaka, Osaka Bay. & EU521642 & EU521643 \\
\hline Porphyra dioica J.Brodie \& L.M.Irvine & $\begin{array}{l}\text { United Kingdom, Sidmouth, WELT A024417 } \\
\text { (nrSSU); WELT A024417 ( } r b c \text { L). }\end{array}$ & HQ687579 & HQ687546 \\
\hline Porphyra linearis Grev. & $\begin{array}{l}\text { United Kingdom, Aberystwyth, BM000898797 } \\
\text { (nrSSU); USA, ME, Kittery, Seapoint, NHA } \\
61204(r b c \text { L). }\end{array}$ & HQ687580 & AF078745 \\
\hline Porphyra lucasii Levring & $\begin{array}{l}\text { Australia, WA, Trigg Beach, WELT A23051 } \\
\text { (nrSSU); WELT-A23051 (rbcL). }\end{array}$ & AY139685 & AY139687 \\
\hline Porphyra purpurea (Roth) C.Agardh & $\begin{array}{l}\text { United Kingdom, Litstock, Somerset, } \\
\text { BM000898974 (nrSSU); BM000898974 (rbcL). }\end{array}$ & HQ687567 & HQ687516 \\
\hline Porphyra umbilicalis Kütz. & $\begin{array}{l}\text { United Kingdom, Sidmouth, WELT A024414 } \\
\text { (nrSSU); WELT A024414 ( } r b c \mathrm{~L}) \text {. }\end{array}$ & HQ687584 & HQ687559 \\
\hline $\begin{array}{l}\text { Pyropia acanthophora (E.C.Oliveira \& } \\
\text { Coll) M.C.Oliveira et al. }\end{array}$ & Brazil, São Paulo, Ubatuba. & L26197 & HQ605695 \\
\hline Pyropia abbottiae (Krishnam) S.C.Lindstr. & $\begin{array}{l}\text { Canada, BC, Victoria, Harling Point, } 25 \text { April } \\
\text { 2005, UBC A85149 (nrSSU); SCL } 12567 \text { ( } r b c \mathrm{~L}) \text {. }\end{array}$ & GU319835 & EU223024 \\
\hline $\begin{array}{l}\text { Pyropia aeodis (N.J.Griffin et al.) J.E. } \\
\text { Sutherland }\end{array}$ & South Africa, Paternoster. & AY292624 & GU165843 \\
\hline Pyropia brumalis (Mumford) S.C. Lindstr. & $\begin{array}{l}\text { Canada, BC, Vancouver, Stanley Park, } 23 \\
\text { February 1998, UBC A84426 (nrSSU); UBC } \\
\text { A84426 ( } r b c \text { L). }\end{array}$ & GU319837 & EU223038 \\
\hline Pyropia californica J.Agardh & $\begin{array}{l}\text { USA, AK, Northeast Ushagat Island, } 25 \text { August } \\
\text { 2006, UBC A86168 (nrSSU); SCL } 13101 \text { (rbcL). }\end{array}$ & GU319849 & EU223117 \\
\hline Pyropia cf. thuretii & $\begin{array}{l}\text { Mexico, Sinaloa, Mazatlán, Olas Altas, WELT } \\
\text { A024423 (nrSSU); WELT A024423 }(r b c \text { L). }\end{array}$ & HQ687587 & HQ687519 \\
\hline $\begin{array}{l}\text { Pyropia cinnamomea (W.A.Nelson) } \\
\text { W.A.Nelson }\end{array}$ & New Zealand, South I, Otago, Bruce's Rock. & AH008010 & EU521637 \\
\hline
\end{tabular}


TABLE 1 (continued)

\begin{tabular}{|c|c|c|c|}
\hline Taxon & Collection data & GenBank A & cession Numbers \\
\hline Pyropia columbina (Mont.) W.A. Nelson & $\begin{array}{l}\text { Falkland Islands, East Falkland, Top Island, Port } \\
\text { Stanley, February 2001, WELT A023553 (nrSSU); } \\
\text { WELT A023553 (rbcL). }\end{array}$ & GU046398 & GU046423 \\
\hline $\begin{array}{l}\text { Pyropia conwayae (S.C.Lindstr. \& } \\
\text { K.M.Cole) S.C.Lindstr. }\end{array}$ & $\begin{array}{l}\text { Canada, BC, French Beach, } 12 \text { March 2007, UBC } \\
\text { A86515 (nrSSU); SCL } 13109(r b c \mathrm{~L}) \text {. }\end{array}$ & GU319838 & EU223045 \\
\hline $\begin{array}{l}\text { Pyropia dentata (Kjellm.) N.Kikuchi \& } \\
\text { M.Miyata }\end{array}$ & $\begin{array}{l}\text { Japan, Chiba, Shirahama, WELT A024400 } \\
\text { (nrSSU); WELT A024400 ( } r b c \text { L). }\end{array}$ & HQ687588 & HQ687520 \\
\hline $\begin{array}{l}\text { Pyropia denticulata (Levring) J.A.Phillips } \\
\text { \& J.E.Sutherland. }\end{array}$ & $\begin{array}{l}\text { Australia, Queensland, Mooloolaba, WELT } \\
\text { A023224 (nrSSU); WELT A023224 (rbcL). }\end{array}$ & HQ687570 & HQ687521 \\
\hline $\begin{array}{l}\text { Pyropia fallax (S.C.Lindstr. \& K.M.Cole) } \\
\text { S.C. Lindstr. }\end{array}$ & $\begin{array}{l}\text { USA, Alaska, Avatanak Island, } 12 \text { June 2008, } \\
\text { UBC A87380 (nrSSU); UBC A87380 ( } r b c \mathrm{~L}) \text {. }\end{array}$ & GU319840 & GU319865 \\
\hline Pyropia fucicola (Krishnam. S.C.Lindstr. & $\begin{array}{l}\text { USA, Alaska, Japonski Island, Sitka airport, SW } \\
\text { side, } 29 \text { end of runway, } 19 \text { August 2005, UBC } \\
\text { A87689 (nrSSU); SCL } 12715(r b c L) \text {. }\end{array}$ & GU319841 & EU223088 \\
\hline Pyropia gardneri & $\begin{array}{l}\text { Mexico, Baja California, Caminitos, Cabo Punta } \\
\text { Banda, CMMEX } 4355 \text { (nrSSU); WELT A024420 } \\
(r b c L) \text {. }\end{array}$ & DQ084423 & HQ687522 \\
\hline Pyropia haitanensis & Japan, Ehime, Yuge. & AB013181 & AB118585 \\
\hline $\begin{array}{l}\text { Pyropia hiberna (S.C.Lindstr. \& } \\
\text { K.M.Cole) S.C.Lindstr. }\end{array}$ & $\begin{array}{l}\text { USA, CA, Monterey County, Pacific Grove, } 31 \\
\text { December 2007, UBC A87474 (nrSSU); UBC } \\
\text { A } 87474(r b c \text { L). }\end{array}$ & GU319843 & GU319866 \\
\hline Pyropia hollenbergii & $\begin{array}{l}\text { Mexico, Baja California Sur, Bahía Agua Verde, } \\
\text { WELT A023231 (nrSSU). }\end{array}$ & HQ687589 & AY794401 \\
\hline $\begin{array}{l}\text { Pyropia ishigecola (A.Miura) N.Kikuchi \& } \\
\text { M.Miyata }\end{array}$ & $\begin{array}{l}\text { Japan, Chiba, Katsuura, Yoshio, WELT A023199 } \\
\text { (nrSSU); WELT A023199 (rbcL). }\end{array}$ & HQ687571 & HQ687524 \\
\hline $\begin{array}{l}\text { Pyropia kanakaensis (Mumford) } \\
\text { S.C.Lindstr. }\end{array}$ & $\begin{array}{l}\text { USA, WA, Olympic Peninsula, between Makah } \\
\text { Bay and Sekui, } 31 \text { May 2003, UBC A86491 } \\
\text { (nrSSU); SCL } 10932(r b c \text { L). }\end{array}$ & GU319844 & EU223099 \\
\hline $\begin{array}{l}\text { Pyropia katadae (A. Miura) M.S. Hwang et } \\
\text { al. }\end{array}$ & Korea, Gyeongsangbukdo, Pohang. & HQ728191 & HQ728199 \\
\hline $\begin{array}{l}\text { Pyropia koreana (M.S.Hwang \& I.K.Lee) } \\
\text { M.S.Hwang et al. }\end{array}$ & - & HQ728190 & HQ728198 \\
\hline $\begin{array}{l}\text { Pyropia kinositae (Yamada \& Tak.Tanaka) } \\
\text { N.Kikuchi et al. }\end{array}$ & Japan, Hokkaido, Suttu, Arito. & EU521640 & EU521641 \\
\hline $\begin{array}{l}\text { Pyropia kuniedae (Kurogi) M.S.Hwang \& } \\
\text { H.G.Choi }\end{array}$ & Korea, Sachon, Gyeongsangnamdo, Namhae. & HQ728192 & HQ728200 \\
\hline Pyropia kurogii (S.C.Lindstr.) S.C.Lindstr. & $\begin{array}{l}\text { Japan, Hokkaido, Utoro, WELT A023201 } \\
\text { (nrSSU); WELT A023201 (rbcL). }\end{array}$ & HQ687573 & HQ687526 \\
\hline $\begin{array}{l}\text { Pyropia lacerata (A.Miura) N.Kikuchi \& } \\
\text { M.Miyata }\end{array}$ & $\begin{array}{l}\text { Japan, Chiba, Shirahama, WELT A024399 } \\
\text { (nrSSU); WELT A024399 ( } r b c \text { L). }\end{array}$ & HQ687574 & HQ687527 \\
\hline Pyropia lanceolata & $\begin{array}{l}\text { Mexico, Baja California, Punta Popotla (nrSSU); } \\
\text { USA, California, Monterey County, Spanish Bay, } \\
1 \text { January 2008, UBC A87475 (rbcL). }\end{array}$ & AY909594 & GU319867 \\
\hline Pyropia leucosticta & $\begin{array}{l}\text { United Kingdom, Sidmouth, BM000898751 } \\
\text { (nrSSU). }\end{array}$ & HQ687593 & DQ191359 \\
\hline
\end{tabular}


TABLE 1 (continued)

\begin{tabular}{|c|c|c|c|}
\hline Taxon & Collection data & GenBank Ac & ession Numbers \\
\hline $\begin{array}{l}\text { Pyropia moriensis (Ohmi) N.Kikuchi \& } \\
\text { M.Miyata }\end{array}$ & Japan, Hokkaido, Otaru. & EU521644 & EU521645 \\
\hline $\begin{array}{l}\text { Pyropia onoi (Ueda) N.Kikuchi \& } \\
\text { M.Miyata }\end{array}$ & $\begin{array}{l}\text { Japan, Hokkaido, Mori, WELT A023216 (nrSSU); } \\
\text { WELT A023216 (rbcL). }\end{array}$ & HQ687575 & HQ687529 \\
\hline Pyropia pendula & $\begin{array}{l}\text { Mexico, Baja California Sur, Calerita, CMMEX } \\
4437 \text { (nrSSU); CMMEX } 4437 \text { (rbcL). }\end{array}$ & DQ084430 & HQ687530 \\
\hline Pyropia perforata & $\begin{array}{l}\text { USA, California, Del Norte County, Crescent City } \\
\text { Lighthouse, } 24 \text { May } 2002 \text { (nrSSU); Mexico, Baja } \\
\text { California, Punta Popotla, } 24 \text { February } 1999 \\
(r b c L) \text {. }\end{array}$ & GU319856 & GU046416 \\
\hline $\begin{array}{l}\text { Pyropia pseudolanceolata (Krishnam) } \\
\text { S.C.Lindstr. }\end{array}$ & $\begin{array}{l}\text { Canada, BC, Victoria, Harling Point, } 5 \text { February } \\
\text { 2005, UBC A87696 (nrSSU); SCL } 12538 \text { (rbcL). }\end{array}$ & GU319857 & EU223145 \\
\hline $\begin{array}{l}\text { Pyropia pseudolinearis (Ueda) N.Kikuchi } \\
\text { et al. }\end{array}$ & $\begin{array}{l}\text { Japan, Chiba, Choshi, WELT A024424 (nrSSU); } \\
\text { WELT A024424 ( } r b c \text { L). }\end{array}$ & HQ687590 & HQ687531 \\
\hline $\begin{array}{l}\text { Pyropia pulchella (Ackland et al.) T.J.Farr } \\
\text { \& J.E.Sutherland. }\end{array}$ & $\begin{array}{l}\text { New Zealand, North I, Waihau Bay East, WELT } \\
\text { A024398 (nrSSU); WELT A024398 (rbcL). }\end{array}$ & HQ687591 & HQ687532 \\
\hline $\begin{array}{l}\text { Pyropia rakiura (W.A.Nelson) } \\
\text { W.A.Nelson }\end{array}$ & New Zealand, South I, Kaikoura, Ocean View. & AF136425 & EU521646 \\
\hline $\begin{array}{l}\text { Pyropia saldanhae (Stegenga et al.) } \\
\text { J.E.Sutherland }\end{array}$ & South Africa, Kommetjie. & AY292630 & GU165838 \\
\hline $\begin{array}{l}\text { Pyropia seriata (Kjellm.) N.Kikuchi \& } \\
\text { M.Miyata }\end{array}$ & $\begin{array}{l}\text { Japan, Kumamoto, Hondo, WELT A023206 } \\
\text { (nrSSU); WELT A023206 (rbcL). }\end{array}$ & HQ687576 & HQ687533 \\
\hline $\begin{array}{l}\text { Pyropia smithii (Hollenberg \& I.A.Abbott) } \\
\text { S.C.Lindstr. }\end{array}$ & $\begin{array}{l}\text { Canada, BC, Vancouver Island, Barkley Sound, } \\
\text { Nudibranch Point, } 10 \text { August 2002, UBC A85584 } \\
\text { (nrSSU); UBC A85585 ( } r b c \mathrm{~L}) \text {. }\end{array}$ & GU319861 & EU223224 \\
\hline $\begin{array}{l}\text { Pyropia spiralis (E.C.Oliveira \& Coll) } \\
\text { M.C.Oliveira } \text { et al. }\end{array}$ & Brazil, Espírito Santo, Vila Velha. & AY766360 & HQ605696 \\
\hline Pyropia suborbiculata & USA, NC, Mansonboro Island, 20 May 1996. & HQ728193 & AY028523 \\
\hline $\begin{array}{l}\text { Pyropia tanegashimensis (Shinmura) } \\
\text { N.Kikuchi \& E.Fujiyoshi }\end{array}$ & $\begin{array}{l}\text { Japan, Kagoshima, Tanegashima, Iseki, WELT } \\
\text { A023236 (nrSSU); WELT A023236 ( } r b c \text { L). }\end{array}$ & HQ727887 & HQ687542 \\
\hline Pyropia tenera (Kjellm.) N.Kikuchi et al. & $\begin{array}{l}\text { Japan, Kumamoto, Kawaura, CMNH-BA-1354 } \\
\text { (nrSSU); CMNH-BA-1354 (rbcL). }\end{array}$ & HQ687577 & HQ687543 \\
\hline $\begin{array}{l}\text { Pyropia tenuipedalis (A.Miura) N.Kikuchi } \\
\text { \& M.Miyata }\end{array}$ & Japan, Chiba, Urayasu. & EU521648 & EU521649 \\
\hline Pyropia torta (V.Krishnam) S.C.Lindstr. & $\begin{array}{l}\text { USA, WA, San Juan Island, Vista Mar, } 22 \text { January } \\
\text { 2002, SCL } 13289 \text { (nrSSU); SCL } 13289 \text { (rbcL). }\end{array}$ & GU319863 & EU223236 \\
\hline $\begin{array}{l}\text { Pyropia vietnamensis (Takido et al.) } \\
\text { J.E.Sutherland \& Monotilla }\end{array}$ & $\begin{array}{l}\text { India, Kerala, Thangeseri, WELT A024426 } \\
\text { (nrSSU); WELT A024426 ( } r b c \mathrm{~L}) \text {. }\end{array}$ & HQ687578 & HQ687544 \\
\hline $\begin{array}{l}\text { Pyropia virididentata (W.A.Nelson) } \\
\text { W.A.Nelson }\end{array}$ & New Zealand, Wellington, North I, Lyall Bay. & AF136421 & EU521650 \\
\hline Pyropia yezoensis & Korea, Gyeongsangnamdo, Tongyoung, Galmok. & HQ728189 & HQ728197 \\
\hline Pyropia raulaguilarii sp. nov. ${ }^{\text {a }}$ & $\begin{array}{l}\text { Mexico, Michoacán, Caletilla, } 6 \text { March 2008, } \\
\text { ENCB } 19524 .\end{array}$ & JQ684704 & JQ684700 \\
\hline Pyropia raulaguilarii sp. nov. ${ }^{\text {a }}$ & $\begin{array}{l}\text { Mexico, Michoacán, Carrizalillo, } 4 \text { April 2009, } \\
\text { ENCB } 19523 .\end{array}$ & JQ684705 & JQ684701 \\
\hline
\end{tabular}

..... continued on the next page 
TABLE 1 (continued)

\begin{tabular}{lll}
\hline Taxon & Collection data & GenBank Accession Numbers \\
\hline Wildemania occidentalis (Setch. \& Hus) & Canada, Vancouver Island British Columbia, Cape & GU319851 \\
S.C.Lindstr. & Palmerston, 17 May 2003, UBC A86488 (nrSSU); & \\
& USA, Washington, Clallam County, Olympic & \\
& Peninsula, between Makah Bay and Sekiu, 31 May & \\
& 2003, SCL 10935 $(r b c \mathrm{~L})$. & \\
\hline
\end{tabular}

${ }^{\text {a }}$ Sequences obtained in this work. ${ }^{\mathrm{b}}$ Outgroups.

TABLE 2: Comparative vegetative and reproductive characteristics of Pyropia species.

\begin{tabular}{|c|c|c|c|c|}
\hline Character & P. hollenbergii ${ }^{1}$ & P. pendula ${ }^{l}$ & P. thuretii ${ }^{2}$ & P. raulaguilarii \\
\hline Height (gametophyte thallus) & $15-22 \mathrm{~cm}$ & $25 \mathrm{~cm}$ & $4-17$ & $4-6 \mathrm{~cm}$ \\
\hline Shape of the blade & Linear-lanceolate & Linear-ligulate & lanceolate & lanceolate \\
\hline Width of the blade & $1-7.5 \mathrm{~cm}$ & $0.1-2$ & $2-8 \mathrm{~cm}$ & $1-2 \mathrm{~cm}$ \\
\hline Thalli thickness & $45-60 \mu \mathrm{m}$ & $40-54 \mu \mathrm{m}$ & $20-40 \mu \mathrm{m}$ & $40-45 \mu \mathrm{m}$ \\
\hline Vegetative cells size Diameter/length & $8-14 / 12-30 \mu \mathrm{m}$ & $6-14 / 8-25 \mu \mathrm{m}$ & $4-10 / 8-20 \mu \mathrm{m}$ & $14-17 / 20-22 \mu \mathrm{m}$ \\
\hline Sexuality & Dioecius & Dioecius & Monoecius & Monoecius \\
\hline Packets of spermatangia & 64 & 128 & 64 & 64 \\
\hline Packets of Zygotosporangia & 8 & 8 & 8 & 16 \\
\hline
\end{tabular}

${ }^{1}$ Aguilar-Rosas et al. (2007). ${ }^{2}$ Aguilar-Rosas \& Aguilar-Rosas (2003).

\section{Discussion}

In the Pacific coast of Mexico there are seven species of Pyropia (Aguilar-Rosas et al., 2007, as Porphyra): Py. gardneri (G.M.Sm. \& Hollenb.) S.C.Lindstr., Py. hollenbergii (E.Y.Dawson) J.E.Sutherland et al., Py. lanceolata (Setch. \& Hus) S.C.Lindstr., Py. pendula (E.Y.Dawson) J.E.Sutherland et al., Py. perforata (J. Agardh) S.C.Lindstr., Py. suborbiculata (Kjellm.) J.E.Sutherland et al., and Py. thuretii (Setch. \& E.Y.Dawson) J.E.Sutherland et al. Among them, Py. hollenbergii and Py. pendula are considered endemic to the Gulf of California, while the remaining five are widely distributed from British Columbia, Canada, to Peru. According to Aguilar-Rosas et al. (2007), the thalli of Py. hollenbergii, Py. pendula and Py. lanceolata have a certain morphological and reproductive similarity. However, characters such as the packet number of spermatangia and zygotosporangia, and the length of the thallus, allows to separate these species. Additionally, Py. thuretii is widely distributed to British Columbia, Canada to Pacific Northwest coast of Mexico.

Regarding Py. lanceolata, it is distributed from the U.S.A. to temperate waters of the Pacific coast of Mexico, it has lanceolate thallus and being over $50 \mathrm{~cm}$ length. Py. perforata grows in dense groups, up to $1 \mathrm{~m}$ length, and wide blades lobed or divided and often located from Alaska to Baja California.

Pyropia raulaguilarii is most closely related with Py. hollenbergii and Py. pendula, morphological and reproductive differences between these species are shown on Table 2. The most distinctive features of Pyropia raulaguilarii are the length of the blade, the surface view in the spermatangial portions and the division formulas of the zygotosporangia (Table 2). In Py. thuretii and Py. hollenbergii both species exhibit differences in blade thickness and formulas of zygotosporangia division; whereas in Py. raulaguilarii there are 16 in Py. thuretii there are 32. On the other hand, Aguilar-Rosas \& Aguilar-Rosas (2003) and Aguilar-Rosas et al. (2007) examined several species of Porphyra (Pyropia) from the Pacific coast of Mexico. Their examination revealed that there are other Pyropia species that may be confused with Py. raulaguilarii mentioned here. Table 2 shows the vegetative and reproductive characters of various species of Pyropia and in it observed the 
main differences between species. For example, the gametophyte thallus of $P y$. raulaguilarii reaches a size of 4.0-6.0 cm, while comparatively Py. hollenbergii and $P y$. pendula reaches up to $25 \mathrm{~cm}$ high and both are dioecious. Furthermore, Py. raulaguilarii, is distributed in the Tropical Pacific of Mexico.

The interspecific divergence obtained for the Pyropia Pacific Mexican taxa was comparable to the amount of variation observed in other Bangiales taxa. Broom et al. (2010) recorded $r b c \mathrm{~L}$ interspecific divergence values between $1.15 \%$ and $9.88 \%$, and recorded nrSSU interspecific divergence values between $1.84 \%$ and 8.39\% for Porphyra species recorded in the Falkland Islands. Lindstrom (2008) reported $r b c$ L interspecific values between 1\% and $4.7 \%$ for Porphyra species from Pacific region. Brodie et al. (2007) obtained a pairwise sequence divergence among Py. rosengurttii, Py. olivii (Orfanidis et al.) J.Brodie \& Neefus and Py. leucosticta (Thur.) Neefus \& J.Brodie in their $r b c \mathrm{~L}$ alignment that ranged from $1 \%$ to $11 \%$. Xu et al. (2011) mentioned that Py. yezoensis (Ueda) M.S.Hwang \& H.G.Choi diverged from Py. haitanensis (T.J.Chang \& B.F.Zheng) N.Kikuchi \& M.Miyata by $1 \%$ of $r b c \mathrm{~L}$ genetic divergence value. Several studies applying molecular techniques to identify species in Porphyra (Stiller \& Waaland 1993, Oliveira et al. 1995, Broom et al. 1999, Kunimoto et al. 1999) have revealed considerable interspecies diversity of the nrSSU and $r b c \mathrm{~L}$ regions within this genus and suggest that provides useful characters for species identification.

The molecular phylogeny showed that samples from Carrizalillo and Caletilla (Michoacán) populations are almost identical, confirming that these samples constitute the same taxonomic entity. The phylogenetic analysis also showed that the mentioned Mexican Pacific samples represent a distinctive and well-supported clade segregated from the rest of the Pyropia representatives.

The high level of genetic variation observed between the Pacific Mexican samples (from Michoacán and Baja California Sur) and other Pyropia species support the recognition of these two taxonomic entities as new taxa within the genus Pyropia. Neefus et al. (2002) considered that Bangiales is understudied and that the number of species could be greater than those recognized at the present.

\section{Acknowledgements}

We thank the Instituto Politécnico Nacional, Universidad Autónoma Metropolitana-Izatapalapa and Universidad Michoacana de San Nicolas de Hidalgo for the support granted to this study. The first and second author thanks Comisión de Operación y Fomento de Actividades Académicas del I.P.N. for a fellowship. LEMC thanks to EDI/IPN. AS., JDL and FFP. thanks to Promep-SEP (UAMI/CA/117). We are grateful to the anonymous reviewers whose suggestions improved significantly this manuscript.

\section{References}

Aguilar-Rosas, L.E. \& Aguilar-Rosas, R. (2003). El género Porphyra (Bangiaceae, Rhodophyta) en la costa Pacífico de México. Hidrobiológica 13: 159-164.

Aguilar-Rosas, R., Aguilar-Rosas, L.E., Sánchez-Rodríguez, I., Broom, J.E. \& Nelson, W.A. (2007). Morfología y distribución de Porphyra hollenbergii (Bangiaceae, Rhodophyta) en la costa del Pacífico de México. Revista Mexicana de Biodiversidad 78: 351-357.

Brodie, J. \& Irvine, L.M. (1997). A comparison of Porphyra dioica sp. nov. and P. purpurea (Roth) C. Ag. (Rhodophyta: Bangiophycidae) in Europe. Cryptogamie Algologie 18: 283-297.

Brodie, J., Bartsch, I, Neefus, C., Orfanidis, S., Bray, T. \& Mathieson, A.C. (2007). New insights into the cryptic diversity of the North Atlantic-Mediterranean 'Porphyra leucosticta' complex: P. olivii sp. nov. and P. rosengurttii (Bangiales, Rhodophyta). European Journal of Phycology 42: 3-28. DOI: 10.1080/09670260601043946

Broom, J.E.S., Farr, T.J. \& Nelson, W.A. (2004). Phylogeny of the Bangia flora of New Zealand suggests a southern origin for Porphyra and Bangia (Bangiales, Rhodophyta). Molecular Phylogenetics and Evolution 31: 1197-2007.

Broom, J.E, Jones, W.A., Hill, D.F., Knight, G.A. \& Nelson, W.A. (1999). Species recognition in New Zealand Porphyra using 18S rDNA sequencing. Journal of Applied Phycology 11 (2): 421-428. DOI: 10.1023/A: 1008162825908

Broom, J.E.S., Nelson, E., Farr, W.A. Phillips, T.J. \& Clayton, M. (2010). Relationships of the Porphyra (Bangiales, Rhodophyta) flora of the Falkland Islands: a molecular survey using rbcL and nSSU sequence data. Australian 
Systematic Botany 23: 27-37.

Broom, J.E., Nelson, W.A., Yarish, C., Jones, W.A., Aguilar-Rosas, R. \& Aguilar-Rosas, L.E. (2002). A reassessment of the taxonomic status of Porphyra suborbiculata, Porphyra carolinensis and Porphyra lilliputiana (Bangiales, Rhodophyta) based on molecular and morphological data. European Journal of Phycology 37: 227-235. DOI: $10.1017 /$ S0967026202003566

Felsenstein, J. (1985). Confidence limits on phylogenies: an approach using the bootstrap. Evolution 39: $783-791$.

Freshwater, D.W. \&. Rueness, J. (1994). Phylogenetic relationships of some European Gelidium (Gelidiales, Rhodophyta) species, based on rbcL nucleotide sequence analysis. Phycologia 33: 187-194.

Huelsenbeck, J.P. \& Ronquist, F.R. (2001). MRBAYES: Bayesian inference of phylogeny. Bioinformatics 17: 754-755. DOI:10.1093/bioinformatics/17.8.754

Jia, J.H., Wang, P., Jin, D.M., Qu, X.P., Wang, Q., Li, C.Y., Weng, M.L. \& Wang, B. (2000). The amplification of RAPD markers in diversity detection and variety identification of Porphyra. Acta Botanica Sinica 42: 403-407.

Jones, W.A., Griffin, N.J., Jones, D.T., Nelson, W.A., Farr, T.J. \& Broom, J.E. (2004). Phylogenetic diversity in South African Porphyra (Bangiales, Rhodophyta) determined by nuclear SSU sequence analysis. European Journal of Phycology 39: 197-211. DOI: 10.1080/0967026042000202145

Kuang, M., Wang, S.J., Li, Y., Shen, D.L. \& Tseng, C.K. (1998). RAPD study on some common species of Porphyra in China. Chinese Journal Oceanology and Limnology 16 : 140-146.

Kunimoto, M., Kito, H., Mizukami, Y., Murase, N. \& Levine I. (2003). Molecular features of a defined genetic marker for the determination of the Porphyra tenera lineage. Journal of Applied Phycology 15: 337-343. DOI: 10.1023/ A: 1025182508440

Kunimoto, M., Kito, H., Yamamoto, Y., Cheney, D.P., Kaminishi, Y. \& Mizukami, Y. (1999). Discrimination of Porphyra species based on small subunit ribosomal RNA gene sequences. Journal of Applied Phycology 11: 203-209. DOI: 10.1023/A:1008019409008

Lindstrom, S.C. (2008). Cryptic diversity, biogeography and genetic variation in northeast Pacific species of Porphyra sensu lato (Rhodophyta, Bangiales). Journal of Applied Phycology 20: 951-962. DOI: 10.1007/s10811-008-9313-9

Lindstrom, S.C. \& Fredericq, S. (2003). rbcL gene sequences reveal relationships among north-east Pacific species of Porphyra (Bangiales, Rhodophyta) and a new species, P. aestivalis. Phycological Research 51: 211-224. DOI: 10.1046/j.1440-1835.2003.00312.x

Iitsuka, O., Nakamura, K., Ozaki, A., Okamoto, N. \& Saga, N. (2002). Genetic information of three pure lines of Porphyra yezoensis (Bangiales, Rhodophyta) obtained by AFLP analysis. Fisheries Sciences 68: 1113-1117.

Liu, H.Q, Yu, W.G., Dai, J.X., Gong, Q.H., Shi, X.C. \& Yang, K.F. (2004). Construction of Porphyra yezoensis pure line from protoplasts and its 18S rDNA sequences determination. Journal of Ocean University of China 3: 60-64.

Mateo-Cid, L.E., Mendoza-González, A.C., Gavio, B. \& Fredericq, S. (2005). Grateloupia huertana sp. nov. (Halymeniaceae, Rhodophyta): a peculiar new prostrate species from tropical Pacific Mexico. Phycologia 44: 4-16.

Neefus, C.D., Mathieson, A.C., Klein, A.S., Teasdale, B., Bray, T. \& Yarish, C. (2002). Porphyra birdiae sp. nov. (Bangiales, Rhodophyta): a new species from the northwest Atlantic. Algae 17: 203-216.

Nelson, W.A., Farr, T.J. \& Broom, J.E.S. (2006). Phylogenetic relationships and generic concepts in the red order Bangiales: challenges ahead. Phycologia 45: 249-259.

Niwa, K., Kikuchi, N. \& Aruga, Y. (2005). Morphological and molecular analysis of the endangered species Porphyra tenera (Bangiales, Rhodophyta). Journal of Phycology 41: 294-304. DOI: 10.1111/j.1529-8817.2005.04039.x

Oliveira, M.C., Kurniawan, J., Bird, J.C., Rice, E.L., Murphy, C.A., Sungh, R.K., Gutell \& Ragan, M.A. (1995). A preliminary investigation of the order Bangiales (Bangiophycidae, Rhodophyta) based on sequences of nuclear small-subunit ribosomal RNA genes. Phycological Research 43: 71-79. DOI: 10.1111/j.1440-1835.1995.tb00007.x

Posada, D. \& Crandall, K.A. (1998). Model test: testing the model of DNA substitution. Bioinformatics 14: 817-818. DOI:10.1093/bioinformatics/14.9.817

Qiao, L.X., Weng, M.L., Kong, F.N., Dai, J.X. \& Wang, B. (2007). The application of RSAP marker technique in diversity detection and germplasm identification of Porphyra. Journal of Ocean University of China 37: 951-956.

Rambaut, A. \& Drummond, A.J. (2007). Tracer v1.4. Available from http://beast.bio.ed.ac.uk/Tracer (accessed 25 April 2012).

Saunders, G.W., Strachan, I.M., West, J.A. \& Kraft, G.T. (1996). Nuclear small-subunit ribosomal RNA gene sequences from representatives Ceramiaceae (Ceramiales, Rhodophyta). European Journal of Phycology 31: 23-29. DOI: 10.1080/09670269600651151

Sun, J.W., Jin, D.M., Zhou, C.J., Yang, Q.K., Weng, M.L., Duan, D.L. Xu, P., Ma, J.H. \& Wang, B. (2005). Identification of Porphyra lines (Rhodophyta) by AFLP DNA fingerprinting and molecular markers. Plant Molecular Biology Reporter 23: 251-262.

Sutherland, J., Lindstrom, S., Nelson, W., Brodie, J., Lynch, M., Hwang, M., Choi, H., Miyata, M., Kikuchi, N., Oliveira, M.C., Farr, T. Neefus, C., Mols-Mortensen, A., Milstein, J. \& Miller, K. (2011). A new look at an ancient order: generic revision of the Bangiales. Journal of Phycology 47: 1131-1151. DOI: 10.1111/j.1529-8817.2011.01052.x

Stiller, J.W. \& Waaland, J.R. (1993). Molecular analysis reveals cryptic diversity in Porphyra (Rhodophyta). Journal of 
Phycology 29: 506-517. DOI: 10.1111/j.1529-8817.1993.tb00152.x

Swofford, D.L. (2002). PAUP*: Phylogenetic Analysis Using Parsimony (*and Other Methods), Version 4. Sinauer Associates, Sunderland.

Teasdale, B., West, A., Taylor, H. \& Klein, A. (2002). A simple restriction fragment length polymorphism (RFLP) assay to discriminate common Porphyra (Bangiophyceae, Rhodophyta) taxa from the Northwest Atlantic. Journal of Applied Phycology 14: 293-298. DOI: 10.1023/A:1021180315743

Thompson, J.D., Higgins, D.G. \& Gibson, T.J. (1994). CLUSTAL W: improving the sensitivity of progressive multiple sequence alignment through sequence weighting, positions-specific gap penalties and weight matrix choice. Nucleic Acids Research 22: 4673-4680.

Weng, M.L, Liu, B., Jin, D.M., Yang, Q.K., Zhao, G., Ma, J.H., Xu, P., Duan, D.L. \& Wang, B. (2005). Identification of 27 Porphyra lines (Rhodophyta) by DNA fingerprinting and molecular markers. Journal of Applied Phycology 17 : 91-97. DOI: $10.1007 / \mathrm{s} 10811-005-4845-8$

$\mathrm{Xu}$, P., Yang, 1., Zhu, J., Xu, H. \& Lu, Q. (2011). Analysis of hybridization strains of Porphyra based on $r b c \mathrm{~L}$ gene sequences. Journal of Applied Phycology 23: 235- 241. DOI: 10.1007/s10811-010-9537-3 